\title{
A workflow for accurate metabarcoding using nanopore MinION sequencing
}

\author{
Bilgenur Baloğlu' ${ }^{1}$ (D) | Zhewei Chen ${ }^{2}$ (D) | Vasco Elbrecht ${ }^{1,3}$ (D) | Thomas Braukmann ${ }^{1}$ (D) | \\ Shanna MacDonald ${ }^{1}$ | Dirk Steinke ${ }^{1,4}$ (D)
}

\author{
${ }^{1}$ Centre for Biodiversity Genomics, \\ University of Guelph, Guelph, ON, Canada \\ ${ }^{2}$ California Institute of Technology, \\ Pasadena, CA, USA \\ ${ }^{3}$ Centre for Biodiversity Monitoring, \\ Zoological Research Museum Alexander \\ Koenig, Bonn, Germany \\ ${ }^{4}$ Integrative Biology, University of Guelph, \\ Guelph, ON, Canada

\section{Correspondence} \\ Bilgenur Baloğlu

\section{Funding information} \\ Canada First Research Excellence Fund; \\ University of Guelph Food From Thought \\ Handling Editor: Erica Leder
}

\begin{abstract}
1. Metabarcoding has become a common approach to the rapid identification of the species composition in a mixed sample. The majority of studies use established short-read high-throughput sequencing platforms. The Oxford Nanopore MinION ${ }^{\mathrm{TM}}$, a portable sequencing platform, represents a low-cost alternative allowing researchers to generate sequence data in the field. However, a major drawback is the high raw read error rate that can range from $10 \%$ to $22 \%$.
\end{abstract}

2. To test whether the MinION ${ }^{\mathrm{TM}}$ represents a viable alternative to other sequencing platforms, we used rolling circle amplification (RCA) to generate full-length consensus DNA barcodes for a bulk mock sample of 50 aquatic invertebrate species with at least $15 \%$ genetic distance to each other. By applying two different laboratory protocols, we generated two MinION ${ }^{\mathrm{TM}}$ runs that were used to build error-corrected consensus sequences. A newly developed Python pipeline, ASHURE, was used for data processing, consensus building, clustering and taxonomic assignment of the resulting reads.

3. Our pipeline achieved median accuracies of up to $99.3 \%$ for long concatemeric reads (>45 barcodes) and successfully identified all 50 species in the mock community. The use of RCA was integral for increasing consensus accuracy but was also the most timeconsuming step of the laboratory workflow. Most concatemeric reads were skewed towards a shorter read length range with a median read length of up to 1,262 bp.

4. Our study demonstrates that Nanopore sequencing can be used for metabarcoding, but exploration of other isothermal amplification procedures to improve consensus accuracy is recommended.

\section{KEYWORDS}

bioinformatics pipeline, metabarcoding, Nanopore sequencing, rolling circle amplification

\section{1 | INTRODUCTION}

DNA metabarcoding uses high-throughput sequencing (HTS) of DNA barcodes to assess the species composition of a heterogeneous bulk sample. It has gained importance in fields such as evolutionary ecology
(Lim et al., 2016), food safety (Staats et al., 2016), disease surveillance (Batovska et al., 2018) and pest identification (Sow et al., 2019). Most metabarcoding studies to date have used short-read platforms such as the Illumina MiSeq (Piper et al., 2019). New long-read instruments such as the Pacific Biosciences Sequel platform could improve taxonomic

This is an open access article under the terms of the Creative Commons Attribution License, which permits use, distribution and reproduction in any medium, provided the original work is properly cited.

(c) 2021 The Authors. Methods in Ecology and Evolution published by John Wiley \& Sons Ltd on behalf of British Ecological Society 
resolution (Heeger et al., 2018; Tedersoo et al., 2018) through long high-fidelity DNA barcodes. In particular, long-read nanopore devices are becoming increasingly popular because these devices are low-cost and portable (Menegon et al., 2017).

Nanopore sequencing is based on the readout of ion current changes occurring when single-stranded DNA passes through a protein pore such as alpha-haemolysin (Deamer et al., 2016). Each nucleotide restricts ion flow through the pore by a different amount, enabling base-calling via time-series analysis of the voltage across a nanopore (Clarke et al., 2009). The first commercially available instrument, Oxford Nanopore Technologies' MinION ${ }^{\mathrm{TM}}$, is a portable sequencing platform that can produce long reads (10 kb to $2 \mathrm{Mb}$ reported; Nicholls et al., 2019). The low start-up costs (starting at $\$ 1,000$ US including a small number of supplies) have made this device increasingly popular among scientists working on molecular species identification (Kafetzopoulou et al., 2018; Loit et al., 2019; Parker et al., 2017), disease surveillance (Quick et al., 2015) and wholegenome reconstruction (Loman et al., 2015). However, a major drawback is the high raw read error rate which reportedly ranges from $10 \%$ to 22\% (Jain et al., 2015, 2018; Kono \& Arakawa, 2019; Krehenwinkel et al., 2019; Sović et al., 2016), a concern when investigating withinspecies diversity or the diversity of closely related species.

However, with consensus sequencing strategies, nanopore instruments can also generate high-fidelity reads for shorter amplicons (Pomerantz et al., 2018; Rang et al., 2018; Simpson et al., 2017). Clustering of corresponding reads is accomplished using a priori information such as reference genomes (Vaser et al., 2017), primer indices marking each sample (Srivathsan et al., 2018) or spatially related sequence information, which can be encoded using DNA amplification protocols such as loop-mediated isothermal amplification (LAMP; Mori \& Notomi, 2009) or rolling circle amplification (RCA; McNaughton et al., 2019). RCA is based on the circular replication of single-stranded DNA molecules. A series of such replicated sequences can be used to build consensus sequences with an accuracy of up to 99.5\% (Calus et al., 2018; Li et al., 2016; Volden et al., 2018). The combination of metabarcoding and nanopore sequencing could allow researchers to generate barcode sequence data for community samples in the field, without the need to transport or ship samples to a laboratory. However, so far only a small number of studies have demonstrated the suitability of MinION ${ }^{\top M}$ for metabarcoding using samples of very low complexity, for example, comprising of 6-11 (Voorhuijzen-Harink et al., 2019) or nine species (Krehenwinkel et al., 2019).

In our study, the full-length DNA barcodes [658 bp of cytochrome oxidase I (COI)] from a bulk sample of 50 aquatic invertebrate species were sequenced with Illumina MiSeq and Oxford Nanopore MinION to assess the feasibility of nanopore sequencing for metabarcoding on bulk samples of greater complexity. Nanopore samples were prepared with two modified RCA protocols (Li et al., 2016) and assessed for their ability to generate concatemeric raw reads for consensus error correction. Barcodes from MiSeq were generated from paired-end sequencing of $\mathrm{COI}$ from the same bulk sample and used to assess community coverage. A new Python pipeline, ASHURE, was developed to perform consensus error correction on concatemeric reads, infer the presence of novel haplotype sequences via OPTICS density-based clustering, explore the error profiles of consensus sequences and assess overall community representation in the complex bulk sample (Baloğlu \& Chen, 2021). ASHURE was compared to C3POa (Volden et al., 2018) on the same dataset to assess the performance of our pipeline relative to other workflows for consensus error correction of nanopore reads. This study showed nanopore sequencing is suitable for metabarcoding studies on samples of greater complexity and can produce barcodes with comparable accuracy to MiSeq.

\section{MATERIALS AND METHODS}

\subsection{Mock community preparation}

A mock community of 50 freshwater invertebrates was constructed from specimens collected with kick-nets in Southern Ontario and Germany. Collection details are recorded in the public dataset DS-NP50M (https://doi.org/10.5883/DS-NP50M) on Barcode of Life Data Systems (BOLD, http://www.boldsystems.org, see Ratnasingham \& Hebert, 2007). A small piece of tissue was subsampled from each specimen (Arthropoda: a leg or a section of a leg; Annelida: a small section of the body; Mollusca: a piece of the mantle) and the DNA was extracted in 96-well plates using membrane-based protocols (Ivanova et al., 2006, 2008). The 658 bp barcode region of $\mathrm{COI}$ was amplified using the following thermal conditions: initial denaturation at $94^{\circ} \mathrm{C}$ for 2 min followed by five cycles of denaturation for $40 \mathrm{~s}$ at $94^{\circ} \mathrm{C}$, annealing for $40 \mathrm{~s}$ at $45^{\circ} \mathrm{C}$ and extension for $1 \mathrm{~min}$ at $72^{\circ} \mathrm{C}$; then 35 cycles of denaturation for $40 \mathrm{~s}$ at $94^{\circ} \mathrm{C}$ with annealing for $40 \mathrm{~s}$ at $51^{\circ} \mathrm{C}$ and extension for $1 \mathrm{~min}$ at $72^{\circ} \mathrm{C}$; and a final extension for $5 \mathrm{~min}$ at $72^{\circ} \mathrm{C}$ (Ivanova et al., 2006). The $12.5 \mu \mathrm{l} \mathrm{PCR}$ mixes included $6.25 \mu \mathrm{l}$ of $10 \%$ trehalose, $2.00 \mu \mathrm{l}$ of ultrapure water, $1.25 \mu \mathrm{l}$ 10X PCR buffer [200 mM Tris- $\mathrm{HCl}(\mathrm{pH} 8.4), 500 \mathrm{mM} \mathrm{KCl}$ ], $0.625 \mu \mathrm{l} \mathrm{MgCl}(50 \mathrm{mM}), 0.125 \mu$ l of each primer cocktail (0.01 mM, C_ LepFolF/C_LepFolR (Hernández-Triana et al., 2014) and for Mollusca C_GasF1_t1/GasR1_t1 (Steinke et al., 2016), $0.062 \mu \mathrm{l}$ of each dNTP (10 mM), $0.060 \mu$ of Platinum ${ }^{\circledR}$ Taq Polymerase (Invitrogen) and $2.0 \mu$ of DNA template. PCR amplicons were visualized on a $1.2 \%$ agarose gel E-Gel ${ }^{\circledR}$ (Invitrogen) and bidirectionally sequenced using sequencing primers M13F or M13R and the BigDye ${ }^{\circledR}$ Terminator v3.1 Cycle Sequencing Kit (Applied Biosystems, Inc.) on an ABI 3730xI capillary sequencer following the manufacturer's instructions. $\mathrm{Bi}$ directional sequences were assembled and edited using Geneious 11 (Biomatters). For specimens without a species-level identification, the Barcode Index Number (BIN) system was used to assign each specimen to a species proxy using the patterns of sequence variation at COI (Ratnasingham \& Hebert, 2013). With this approach, a total of 50 OTUs was selected with $15 \%$ or more K2P COI distance (Kimura, 1980) from other sequences for the mock sample. A complete list of specimens, including taxonomy, collection details, sequences, BOLD accession numbers and Nearest Neighbour distances are provided in Table S1. 


\section{2 | Bulk DNA extraction}

The remaining tissue of the mock community specimens was dried overnight, pooled and subsequently placed in a sterile $20 \mathrm{ml}$ tube containing 10 steel beads ( $5 \mathrm{~mm}$ diameter) to be homogenized by grinding at 1,702 $\mathrm{g}$ for 30-90 $\mathrm{min}$ in an IKA ULTRA TURRAX Tube Drive Control System (IKA Works). A total of $22.1 \mathrm{mg}$ of homogenized tissue was used for DNA extraction with the Qiagen DNeasy Blood and Tissue kit (Qiagen) following the manufacturer's instructions. DNA extraction success was verified on a $1 \%$ agarose gel (100 V, $30 \mathrm{~min}$ ) and DNA concentration was quantified using the Qubit HS DNA Kit (Thermo Fisher Scientific).

\section{3 | Metabarcoding using Illumina sequencing}

Bulk DNA extract was amplified using fusion primer-based twostep PCR protocol (Elbrecht \& Steinke, 2019) prior to Illumina sequencing. During the first PCR step, a $421 \mathrm{bp}$ region of the Cytochrome c oxidase subunit I (COI) was amplified using the BF2/ BR2 primer set (Elbrecht \& Leese, 2017). PCRs were carried out in a $25 \mu$ reaction volume, with $12.5 \mathrm{ng}$ DNA, $0.2 \mu \mathrm{M}$ of each primer and $12.5 \mu \mathrm{l} \mathrm{PCR}$ Multiplex Plus buffer (Qiagen). The PCR was carried out in a Veriti thermocycler (Thermo Fisher Scientific) using the following cycling conditions: initial denaturation at $95^{\circ} \mathrm{C}$ for $5 \mathrm{~min} ; 25$ cycles of: $30 \mathrm{~s}$ at $95^{\circ} \mathrm{C}, 30 \mathrm{~s}$ at $50^{\circ} \mathrm{C}$ and $50 \mathrm{~s}$ at $72^{\circ} \mathrm{C}$; and a final extension of $5 \mathrm{~min}$ at $72^{\circ} \mathrm{C} .12 .5 \mathrm{ng}(1 \mu \mathrm{l})$ of PCR product was used as the template for the second PCR, where Illumina sequencing adapters were added using individually tagged fusion primers (Elbrecht \& Steinke, 2019). For the second PCR, the reaction volume was increased to $35 \mu \mathrm{l}$, the cycle number reduced to 20 , and extension times increased to 2 min per cycle. PCR products were purified and normalized using SequalPrep Normalization Plates (Thermo Fisher Scientific, Harris et al., 2010) according to the manufacturer protocols. $10 \mu \mathrm{l}$ of each normalized sample was pooled, and the final library was cleaned using left-sided size selection with 0.76× SPRIselect (Beckman Coulter). Sequencing was carried out by the Advances Analysis Facility at the University of Guelph using a 600 cycle Illumina MiSeq Reagent Kit v3 and 5\% PhiX spike in. The forward read was sequenced for an additional 16 cycles (316 bp read).

The resulting sequence data were processed using the JAMP pipeline v0.67 (github.com/VascoElbrecht/JAMP). Sequences were demultiplexed, paired-end reads merged using Usearch v11.0.667 with fastq_pctid=75 (Edgar, 2010), reads below the read length threshold (414 bp) were filtered and primer sequences trimmed both using Cutadapt v1.18 with default settings (Martin, 2011). Sequences with poor quality were removed using an expected error value of 1 (Edgar \& Flyvbjerg, 2015) as implemented in Usearch. MiSeq reads, including singletons, were clustered using cd-hit-est ( $\mathrm{Li}$ \& Godzik, 2006) with parameters: -b 100 -c 0.95 -n 10. Clusters were subsequently mapped against the mock community data as well as against the BOLD COI reference library.

\subsection{Metabarcoding using Nanopore sequencing}

A modified intramolecular-ligated Nanopore Consensus Sequencing (INC-Seq) approach (Li et al., 2016) that employs rolling circle amplification (RCA) of circularized templates was used to generate linear tandem copies of the template for sequencing. An initial PCR was prepared in $50 \mu \mathrm{l}$ reaction volume with $25 \mu \mathrm{l} 2 \times$ Multiplex PCR Master Mix Plus (Qiagen), 10 pmol of each primer (for 658 bp COI barcode fragment-Table S2), $19 \mu \mathrm{l}$ molecular grade water and $4 \mu \mathrm{l}$ DNA. PCR was performed on a Veriti thermocycler (Thermo Fisher Scientific) with the following cycling conditions: initial denaturation at $98^{\circ} \mathrm{C}$ for $30 \mathrm{~s}, 35 \mathrm{cycles}$ of $\left(98^{\circ} \mathrm{C}\right.$ for $30 \mathrm{~s}, 59^{\circ} \mathrm{C}$ for $30 \mathrm{~s}, 72^{\circ} \mathrm{C}$ for $30 \mathrm{~s})$ and a final extension at $72^{\circ} \mathrm{C}$ for $2 \mathrm{~min}$. Amplicons were purified using SpriSelect (Beckman Coulter) with a sample to volume ratio of $0.6 \times$ and quantified using a High Sensitivity dsDNA Kit on a Qubit fluorometer (Thermo Fisher Scientific). $5 \mu$ of Blunt/TA Ligase Master Mix (NEB) was used to self-ligate $55 \mu$ of purified amplicons at a concentration of $2-3 \mathrm{ng} / \mu \mathrm{l}$ into plasmid like structures. Products were subsequently treated with the Plasmid-Safe ${ }^{\text {TM }}$ ATP-dependent DNAse kit (Lucigen Corp) to remove remaining linear molecules. Final products were again purified with SpriSelect at a $0.6 \times$ ratio and quantified using the High-Sensitivity dsDNA Kit on a Qubit fluorometer (Thermo Fisher Scientific). Rolling Circle Amplification (RCA) was performed for six $2.5 \mu$ aliquots of circularized DNA with 0.3-0.4 $\mathrm{ng} / \mu \mathrm{l}$ starting concentration plus negative controls (water) using the TruePrime ${ }^{\mathrm{TM}}$ RCA kit (Expedeon Corp) following the manufacturer's instructions. RCA products were incubated for 1-6 hr at $30^{\circ} \mathrm{C}$. RCA was stopped once $60-70 \mathrm{ng} / \mu \mathrm{l}$ of double-stranded DNA was reached, which corresponded to around 5-6 hr of incubation. Subsequently, RCA products were incubated for $10 \mathrm{~min}$ at $65^{\circ} \mathrm{C}$ to inactivate the enzyme. Two experiments were performed under varying RCA conditions (Protocols A and B, detailed in Table 1), such as RCA duration (influences number of RCA fragments), fragmentation duration and fragmentation methods. Protocol A followed $\mathrm{Li}$ et al. (2016) by incubating $65 \mu \mathrm{l}$ of pooled RCA product with $2 \mu \mathrm{l}(20$ units) of T7 Endonuclease I (NEB, M0302S, VWR Canada) at room temperature for 5 min of enzymatic debranching, followed by mechanical shearing using a Covaris g-TUBE ${ }^{\mathrm{TM}}$ (D-Mark Biosciences) at $1,877 \mathrm{~g}$ for $1 \mathrm{~min}$ on each side of the tube or until the entire reaction mix passed through the fragmentation hole. Protocol $B$ is a modified

TABLE 1 Varying rolling circle amplification (RCA) conditions for experimental protocols $\mathrm{A}$ and $\mathrm{B}$

\begin{tabular}{|lll|}
\hline Dataset & Protocol A & Protocol B \\
\hline RCA duration (hr) & 5 & 6 \\
\hline $\begin{array}{l}\text { Number of target } \\
\text { sequences per RCA } \\
\text { fragment }\end{array}$ & 12 & 15 \\
\hline $\begin{array}{l}\text { Enzymatic branching (min) } \\
\text { Mechanical fragmentation }\end{array}$ & 5 & $2,877 \mathrm{~g}, 2$ min \\
\hline Primer pairs used & $\begin{array}{l}\text { HCOA-LCO, } \\
\text { HCOC2-LCOC2 }\end{array}$ & $\begin{array}{l}\text { HCOA2-LCOA2, } \\
\text { HCOC2-LCOC2 }\end{array}$ \\
\hline
\end{tabular}


approach to counteract the overaccumulation of smaller DNA fragments, where only 2 min of enzymatic debranching was applied with no subsequent mechanical fragmentation. To verify the size of fragments after shearing, sheared products for both protocols were run on a $1 \%$ agarose gel at $100 \mathrm{~V}$ for $1 \mathrm{hr}$. DNA damage was repaired by incubating $53.5 \mu \mathrm{l}$ of the product with $6.5 \mu \mathrm{l}$ of FFPE DNA Repair Buffer and $2 \mu$ l of NEBNext FFPE Repair mix (VWR Canada) at $20^{\circ} \mathrm{C}$ for 15 . The final product was purified using SpriSelect at a $0.45 \times$ ratio and quantified using a Qubit fluorometer.

For sequencing library preparation, Nanopore Genomic Sequencing Kit SQK-LSK308 (Oxford Nanopore) was used. First, the NEBNext Ultra II End Repair/dA Tailing kit (NEB) was used to end repair $1,000 \mathrm{ng}$ of sheared RCA product (1 microgram of DNA in $50 \mu$ l nuclease-free water, $7 \mu$ l of Ultra II End-Prep Buffer, $3 \mu$ I Ultra II End-Prep Enzyme Mix in a total volume of $60 \mu \mathrm{l})$. The reaction was incubated at $20^{\circ} \mathrm{C}$ for $5 \mathrm{~min}$ and heat inactivated at $65^{\circ} \mathrm{C}$ for another $5 \mathrm{~min}$. The resulting DNA was purified using SpriSelect at a 1:1 ratio according to the SQK-LSK308 protocol. Then it was eluted in $25 \mu \mathrm{l}$ of nuclease-free water and quantified with a recovery aim of $>70 \mathrm{ng} / \mu \mathrm{l}$. Blunt/TA Ligase Master Mix (NEB) was used to ligate native barcode adapters to $22.5 \mu \mathrm{l}$ of $500 \mathrm{ng}$ end-prepared DNA at room temperature (10 min). DNA was purified using a 1:1 volume of SpriSelect beads and eluted in $46 \mu \mathrm{l}$ nuclease-free water before the second adapter ligation. For each step, the DNA concentration was measured. The library was purified with $A B B$ buffer provided in the SQK-LSK308 kit (Oxford Nanopore). The final library was then loaded onto a MinION flow cell FLO-MIN107.1 (R9.5) and sequenced using the corresponding workflow on MinKNOW ${ }^{\mathrm{m}}$. Basecalling was performed using Guppy 3.2.2 in CPU mode with the dna_r9.5_450bps_1d2_raw.cfg model.

\section{5 | ASHURE data processing workflow}

The following is a brief overview of the ASHURE (A Safe Heuristic Under Random Events) pipeline (Baloğlu \& Chen, 2021). More details on statistical measures and parameters used are found in the Supplemental Material (Methods S1 to S2.3, the flowchart of the workflow is found in Figures S1 and S2).

\subsection{1 | Pseudo-reference database generation}

The ASHURE pipeline uses pseudo-reference sequences to find concatemers in the raw reads. The pseudo-reference database was generated by searching a subset of raw reads for subsequence windows containing both forward and reverse primers.

\subsection{2 | Concatemer identification}

Concatemers were identified by mapping each raw read against the pseudo-reference database with minimap2 (Li, 2018). Putative concatemers were sorted by the alignment score. Only the highestscoring non-overlapping alignments in each raw read were kept for downstream analysis.

\subsection{3 | Consensus error correction}

For raw reads with more than one concatemer, concatemers were extracted, reoriented $5^{\prime}->3^{\prime}$ and multi-aligned with spoa (Vaser et al., 2017) to generate an error-corrected consensus sequence for each read.

\subsection{4 | Primer identification}

Error-corrected reads were mapped to forward and reverse primer sequences with minimap2. Primer pairs were assigned based on the highest combined alignment score.

\subsection{5 | Clustering}

The OPTICS algorithm (Ankerst et al., 1999) was used to perform clustering on error-corrected reads to infer the true haplotype sequences. On an OPTICS reachability plot (Figures S4 and S6), the region with the lowest reachability for a given cluster represents the center of the cluster. Cluster centers are consensus sequences computed from a multi-alignment of sequences in these low reachability regions and match closely with the true haplotype sequence.

\subsection{6 | Data visualization}

t-SNE, t-distributed stochastic neighbour embedding (Maaten \& Hinton, 2014), was used to perform dimensionality reduction on pairwise distances computed from sequence data to portray the relationship between sequences on a 2D plot.

\subsection{7 | Statistical analysis}

Correlation coefficients were determined in ASHURE using the Numpy (Walt et al., 2011) and PANDAs packages (McKinney, 2010).

\section{6 | Comparison with C3POa}

R2C2's (Rolling Circle Amplification to Concatemeric Consensus) post-processing pipeline C3POa (Concatemeric Consensus Caller using partial order alignments) was used to generate $\mathrm{R} 2 \mathrm{C} 2$ errorcorrected reads from protocols $A$ and $B$ base called fastq data following instructions in Volden et al. (2018). Haplotype matching and 
error profiling for each R2C2 consensus read were computed with get_accuracy.py per Supplementary Materials and Methods Section S2.3. Unlike ASHURE, C3POa does not report information on the concatemer length; hence, direct comparisons for different thresholds were not possible.

\section{3 | RESULTS}

\section{1 | Mock community}

Many collected specimens could not be readily identified to species level. Consequently, the Barcode Index Number (BIN) system, which examines patterns of sequence variation at $\mathrm{COI}$, was used to assign each specimen to a species proxy (Ratnasingham \& Hebert, 2013). In all, 50 BINs showing $>15 \%$ COI sequence divergence from their nearest neighbour under the Kimura 2-parameter model (Kimura, 1980) were retrieved. The freshwater macrozoobenthos mock community included representatives of 3 phyla, 12 orders and 27 families. COI sequences have been deposited on NCBI Genbank under the Accession Numbers MT324068-MT324117. Further specimen details can be found in the public dataset DS-NP50M (https://doi. org/10.5883/DS-NP50M) on BOLD.

\section{2 | Metabarcoding using Illumina sequencing}

Illumina MiSeq sequencing generated an average of 204,797 pairedend reads per primer combination. In all, 49 out of 50 OTUs present in our mock community (Figure 1d) were recovered. Overall, 845 OTUs were detected by clustering. These OTU consisted mostly of contaminants that were also present in nanopore sequencing. An OTU table including sequences, read counts and assigned taxonomy is available as Table S3.

\subsection{Metabarcoding using Nanopore sequencing}

Nanopore sequencing with the MinION delivered 746,153/2,756 and $499,453 / 1,8741 D / 1 D^{2}$ reads for Protocols $A$ and B (SRA PRJNA627498), respectively. The 1D approach only sequences one template DNA strand, whereas with the $1 D^{2}$ method both complementary strands are sequenced, and the combined information is used to create a higher-quality consensus read (Cornelis et al., 2019). Because of the low read output for $1 D^{2}$ reads, our analyses focused on 1D data. Most reads were skewed towards a shorter read length range (Figure 2) with a median RCA fragment length of 1,262 bp for Protocol A and 908 bp for Protocol B.
ASHURE Protocol A


FIGURE 1 Nanopore sequencing read error per species for (a) Protocol A and (b) Protocol B obtained with ASHURE using all reads. (c) Nanopore sequencing read error obtained with OPTICS in ASHURE using cluster centers for each rolling circle amplification (RCA) condition. (d) MiSeq sequencing read error per species. The scale on the $y$ axis differs between the figures 
With flexible filtering (number of targets per RCA fragment $=1$ or more), ASHURE provided a median accuracy of $92.16 \%$ for Protocol A and $92.87 \%$ for Protocol B (see Table 2; Figure 1a,b). For both protocols, a negative non-significant correlation between consensus median error and the number of RCA fragments (Pearson's $r$ for Protocol A: -0.247, Protocol B: -0.225) was observed. A positive non-significant correlation between consensus median error and primer error (Pearson's $r$ for Protocol A: 0.228, Protocol B: 0.375) and between consensus median error and cluster centre error (see Figure 3b,c; Pearson's $r$ for Protocol A: 0.770, Protocol B: 0.274) was observed. One-fifth of the OTUs in Protocol A and half of the OTUs in Protocol B had median accuracy values $>95 \%$. Increasing the number of RCA fragments to 15 or more came with the tradeoff of detecting fewer OTUs (from 50 to 36 for Protocol A and 50

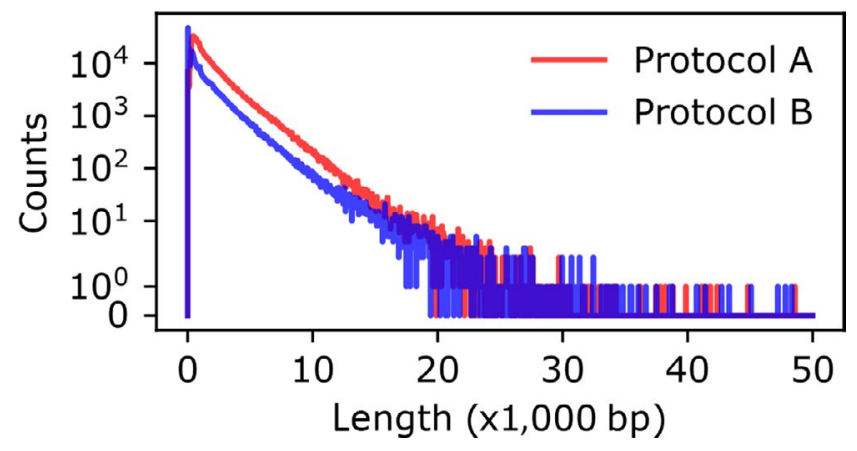

FIGURE 2 Read length distribution for both sequencing protocols. The number of reads is provided on a logarithmic scale on the $y$-axis to 38 for Protocol B). At the same time, median accuracy values increased to $97.4 \%$ and $97.6 \%$ for Protocols $A$ and B, respectively. With more stringent filtering (number of targets per RCA fragment $=45$ or more), median accuracy improved up to $99.3 \%$ for both Protocols $A$ and $B$ but with the trade-off of an overall reduced read output and $a$ reduced number of species recovered (Table 2 ).

The 845 OTUs found in the MiSeq dataset were used to exclude contaminants $(69,911$ for Protocol A and 31,045 reads for Protocol B) in ASHURE results. With Miseq, 49 out of 50 of the mock species were detected, whereas all 50 mock community species were detected in both nanopore sequencing protocols $A$ and $\mathrm{B}$. Using the MiSeq dataset, contaminants from the consensus reads obtained with C3POa (8,843 for Protocol A and 4,222 reads for Protocol $B$ ) were also removed. C3POa produced fewer consensus reads than ASHURE for Protocol B (see Table 2), but the median consensus accuracy using flexible filtering was similar (94.5\%-94.7\% Protocols A and B). The median accuracy when including all consensus reads was higher for C3POa than ASHURE in both Protocols A and B. Overall, the two pipelines showed similar performance in consensus read error profile (Figures S10a-d and S11). As for Protocol B, ASHURE detected a higher number of mock community species (see Table 2).

Although the error profile for all error-corrected consensus reads (Figure 1a,b) spanned a wide range (0\%-10\% error), running OPTICS, a density-based clustering algorithm, on the error-corrected reads enabled us to identify high-fidelity cluster centres (Figure 1c), which possessed comparable accuracy to MiSeq (Figure 1d). Compared to RCA length and UMI error, cluster centre error correlated strongly

TABLE 2 Consensus reads, median accuracy and the number of OTUs/species detected at different thresholds for Protocol A and B analysed with ASHURE and C3POa

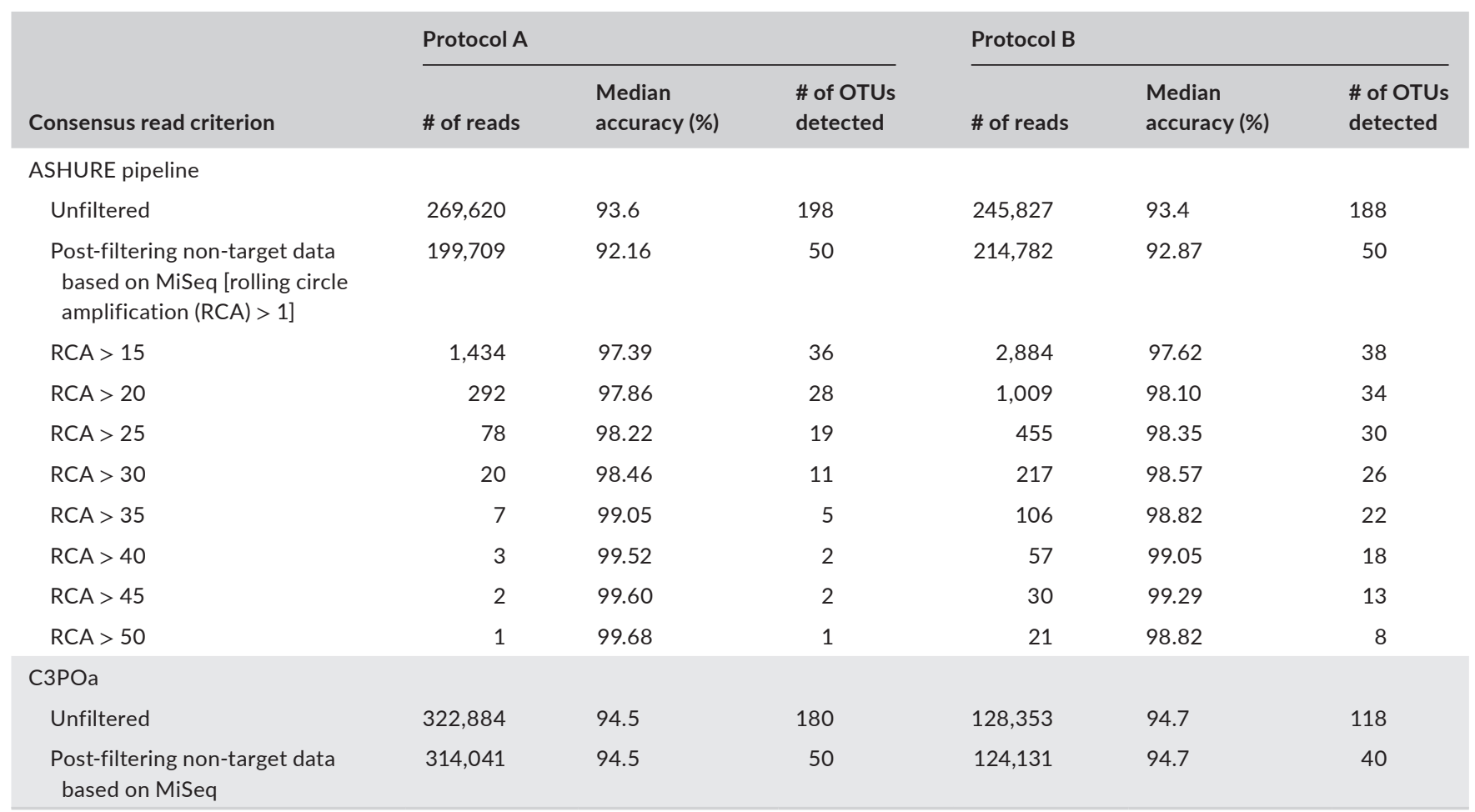



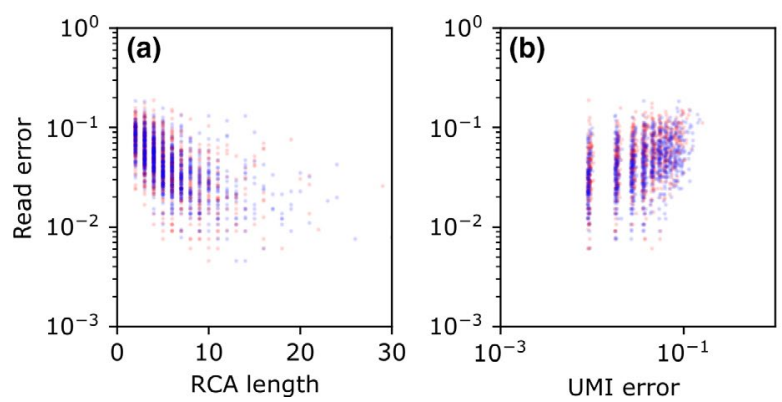

FIGURE 3 Comparison of consensus error versus (a) rolling circle amplification (RCA) length, (b) UMI error, and (c) cluster center error using the ASHURE pipeline for two RCA conditions (a)

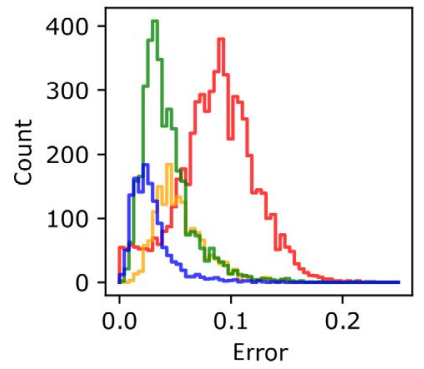

(b) All samples

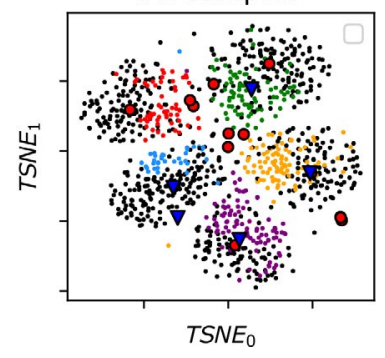

(c)

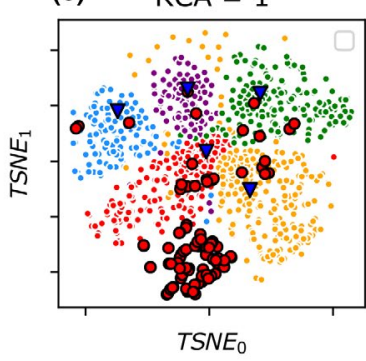

(e) $\mathrm{RCA}=5-8$

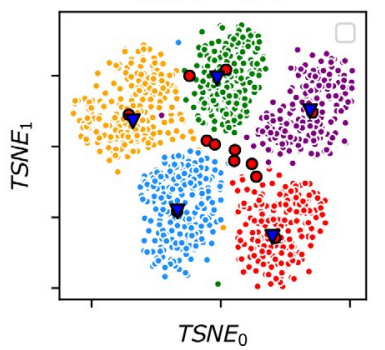

(d) $\mathrm{RCA}=2-4$

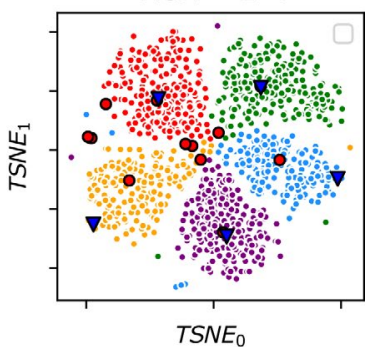

(f) RCA $=9$ and above

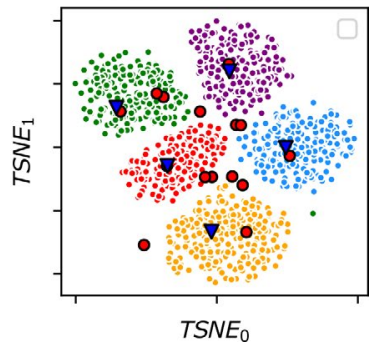

FIGURE 4 tSNE visualization of reference-free clustering using OPTICS for five randomly selected haplotypes. (a) The number of reads and percentage of error for each filtering criteria, red: reads with 1 rolling circle amplification (RCA) fragment, yellow: reads with 2-4 RCA fragments, green: reads with 5-8 RCA fragments, and blue: reads with 9 or more RCA fragments. tSNE visualization of OPTICS clusters for reads with (b) no filtering, (c) one RCA fragment, (d) 2-4 RCA fragments, (e) 5-8 RCA fragments, (f) 9 and more RCA fragments. True haplotypes (blue triangles) and cluster centers obtained with reference-free clustering (red circles) overlap more as the number of RCA fragments increases. Colors in b-f correspond to HAP04 (red), HAP11 (blue), HAP17 (purple), HAP39 (orange), HAP41 (green). Grey dots in (b) indicate outliers

with consensus read error, particularly for Protocol A (Pearson's $r$ : 0.770; see Figure $3 a-c$ ), which means cluster centres closely matched the haplotype sequences present in the mock sample. To visualize why OPTICS can identify high-fidelity cluster centres, five OTUs were randomly selected and clustered at different RCA fragment lengths (Figure 4). T-distributed stochastic neighbour embedding (t-SNE) was used to visualize the co-similarity relationship of these sequences in two dimensions (Figure 4b-f). Closely related sequences clustered together and corresponded to the OTUs obtained by OPTICS. Clustering of raw reads resulted in less informative clusters, where OTUs were not well separated and cluster membership did not always match that of the true species (Figure 4c). The clustering of reads with increasing RCA length cut-off resulted in clusters that had more distinct boundaries (Figure 4d-f), illustrating the increasing relative genetic distance between unassociated reads as higher-fidelity reads were retained. These cluster labels matched their species identity (Figure $4 \mathrm{f}$ ) and encapsulated the de novo cluster centres and true OTU sequences at their centroids. The OPTICS algorithm successfully extracted the OTU structure embedded in a co-similarity matrix, flagged low-fidelity reads that were in the periphery of each cluster, and assigned high-fidelity reads to the centre of the clusters (Figure $4 \mathrm{~b}$ ).

\section{DISCUSSION}

This study demonstrated the feasibility of bulk sample metabarcoding with nanopore devices by generating high-fidelity barcodes through consensus error correction of concatemeric reads and performing reference-free species identification with OPTICS densitybased clustering. Despite the successes of our workflow, notable trade-offs, such as read coverage and accuracy, exist. The RCA protocol used was not efficient in generating long concatemeric reads, and optimizations to this protocol must be tailored to the study 
being conducted. Our pipeline performed well relative to existing pipelines such as C3POa, but this suggests both methods have hit an information bottleneck. The utility of our novel density-based clustering approach for reference-free species identification also requires more discussion. Our suggestions for further improvements to the molecular and bioinformatics workflow are discussed below.

Our study was able to confidently map each read to their haplotype groups because each species in the mock community had at least $15 \%$ genetic distance to each other. This would likely change if a sample included some species that were more closely related $(<3 \%$ genetic distance). In fact, most community samples are a mixture of species with varying genetic distances. In these cases, stringent filtering for reads with more concatemers, resulting in high consensus accuracy (see Figure 3a), would be required so each read can be confidently mapped to their haplotype groups. However, aggressive RCA length filtering can result in loss of coverage for rare species. A trade-off between median consensus accuracy and detection of rare species was observed in our study (see Table 2; Figure 2) because some rarer haplotypes simply do not have many long concatemeric reads.

The distribution of concatemer lengths is strongly influenced by the RCA protocol and optimizing the protocol to enrich a sample with longer concatemeric reads was done to help mitigate this accuracy versus species coverage trade-off. Although both of our RCA protocols were successful in generating concatemers, most reads were still skewed towards single copy COI, suggesting RCA was not efficient at enriching a sample with long concatemers. Overall, protocol B had a slightly greater fraction of long concatemeric reads, higher detected species and higher median accuracy. Protocol B used a higher number of RCA replicates as input DNA, had no mechanical fragmentation step and a reduced duration of enzymatic debranching (Table 1). This workflow seems to be more suitable for situations where stringent concatemer length filtering is used, but more replicates are still required to establish a firm conclusion. For studies where a low error rate is of utmost importance, the fraction of long concatemeric reads in the sample could be further improved with size selection, increasing the RCA incubation time or performing multiple nanopore runs and pooling reads. However, these optimizations are not necessarily suitable for time-sensitive studies such as those conducted in the field. Although RCA was integral to our consensus error-correction workflow, it was also the most time-consuming laboratory step, for example, 5-6 hr of amplification was needed to generate enough product $(60-70 \mathrm{ng} / \mu \mathrm{l})$ for sequencing. For field-based studies, the RCA duration should be adjusted based on the expected complexity of the sample.

Given some of the weaknesses in RCA, exploration of other isothermal amplification procedures such as LAMP (Mori \& Notomi, 2009; Shepherd et al., 2020), multiple displacement amplification (Hansen et al., 2018) or recombinase polymerase amplification (Donoso \& Valenzuela, 2018) is recommended. ASHURE is not limited to the RCA workflow and can be adapted for these other protocols. ASHURE relies on pseudo-reference sequences to identify concatemeric reads and paired-end primer searches are used to establish this pseudo-reference database. The pipeline can be used to process outputs of other isothermal amplification methods generating concatenated molecules by simply providing primer/UMI sequences that link each repeating or alternately repeating segment.

Previous studies using circular consensus approaches to Nanopore sequencing, such as INC-seq (Li et al., 2016) and R2C2 (Volden et al., 2018) have already shown improvements in read accuracy. C3POa, the post-processing pipeline for R2C2, reported a median accuracy of $94 \%$ (Volden et al., 2018). When our pipeline ASHURE was compared to C3POa, only modest differences in read coverage (Figure S10) and accuracy (Figure S11) were observed. ASHURE produced more consensus reads than C3POa (see Table 2), but this is likely due to different parameters applied in ASHURE and C3POa, that is, C3POa data processing includes the detection of DNA splint sequences and the removal of short $(<1,000 \mathrm{~kb})$ and lowquality $(Q<9)$ reads (Volden et al., 2018), whereas in ASHURE this preprocessing step does not exist. With C3POa, a raw read is only used for consensus calling if one or more specifically designed splint sequences are detected within it (Volden et al., 2018). In ASHURE, primer sequences were used to identify reads for further consensus assembly instead of splint sequences. Our approach avoids Gibson assembly, an additional step in the R2C2 workflow. Both C3POa and ASHURE showed similar accuracy for our datasets, but C3POa detected fewer species in our Protocol B experiment. In ASHURE, only $43.4 \%$ and $7 \%$ of the reads in Protocols $A$ and $B$, respectively, contained both forward and reverse primer pairs. This suggests RCA preferentially amplifies short fragmented DNA rather than fulllength $\mathrm{COI}$. This may explain why C3POa generated fewer consensus reads in Protocol B, as the number of detected sequences was very low. Initially, increasing the unique molecular identifier (UMI) length for our primers was considered useful not only for consensus calling but also for identifying, quantifying and filtering out lowfidelity reads. However, within the small percentage of reads with both primers attached, no strong correlation was found between the $\mathrm{UMI}$ error and the consensus read error (Figure $3 \mathrm{~b}$ ). Since C3POa performs so similarly to ASHURE, these respective methods must have hit an information bottleneck because both methods rely on the same underlying multi-alignment procedure for error correction. Further improvements to these types of pipelines could only be gained by leveraging newer base-calling algorithms that examine the raw signal in fast 5 files.

Reference-free approaches for barcode generation with MinION ${ }^{\mathrm{TM}}$ are important for weaning off our dependence on Sanger and Illumina technologies for high-fidelity sequence information. Studies implementing a reference-free approach primarily use tagged amplicon sequencing, which allows for sequence-to-specimen association (Calus et al., 2018; Pomerantz et al., 2018; Srivathsan et al., 2018, 2019). These approaches are useful for species-level taxonomic assignment (Benítez-Páez et al., 2016) and even species discovery (Srivathsan et al., 2019). Our pipeline achieves referencefree barcode generation with density-based clustering, which is a promising approach for examining species diversity in mixed samples. The density-based clustering of Nanopore reads allows for a reference-free approach by grouping reads with their replicates without having to map to a reference database (Faucon et al., 2017). 
Conventional OTU threshold clustering approaches are not suitable for nanopore data. Either each sequence was assigned to a unique OTU or the OTU assignment failed due to the variable error profile (Ma et al., 2017). The optimal threshold depends on the relative abundance of species in a given sample (Mafune et al., 2019). Density-based clustering is advantageous because cluster boundaries are adaptively determined based on other objects in the neighbourhood (Ankerst et al., 1999). The resulting clusters tend to be more parsimonious than thresholding approaches. Clusters correspond to regions in which the data points are densely packed, and noise is regarded as regions of low object density (Ankerst et al., 1999). For DNA sequences, this clustering approach requires sufficient coverage around a true amplicon so that the novel clusters can be detected and are not treated as noise. With sufficient read counts, density-based approaches allow us to obtain any possible known or novel species cluster with high accuracy and without the need for a reference database (see Figure 4f).

Although nanopore sequencing was shown to be capable of producing high-fidelity barcodes, the Illumina MiSeq platform is still a useful complement to nanopore sequencing. In our study, MiSeq results were used to identify potential off-target amplification by-products or contaminants present in the bulk sample. This information was used to exclude erroneous nanopore reads unassociated with any of the mock 50 haplotypes. Although the MiSeq experiment detected one species less than in the Nanopore experiment, these differences could be explained by primer bias because a different primer set was used for MiSeq paired-end barcode retrieval. In terms of accuracy, the MiSeq platform performs slightly better (Figure 1c,d), but the reduced error rates and longer barcodes generated by the MinION ${ }^{\mathrm{TM}}$ make it a more cost-efficient alternative. In the absence of complementary MiSeq data, potential contaminants in a bulk sample could also be identified using a reference database such as the Barcode of Life Data system (Ratnasingham \& Hebert, 2007), which highlights the importance of building such high-fidelity databases.

\section{5 | CONCLUSIONS}

This study demonstrates the feasibility of bulk sample metabarcoding with Oxford Nanopore sequencing using a modified molecular and novel bioinformatics workflow. Our workflow was able to obtain high-fidelity COI barcodes with median accuracies of up to $99.3 \%$ and implement a novel reference-free approach to barcode generation with OPTICS density-based clustering. This study was based on aquatic invertebrates, but the pipeline can be extended to many other taxa and ecological applications. Our workflow is not perfect. Highly accurate results are possible, but with the trade-off in the number of species that can be detected. The exploration of other isothermal amplification techniques and error-correction methods based on the raw signal are recommended. Although nanopore sequencing cannot fully replace MiSeq in metabarcoding studies, this technology is still a very promising and cost-efficient alternative for future bioassessment programs.

\section{ACKNOWLEDGEMENTS}

We thank all staff at the Centre for Biodiversity Genomics who helped to collect the samples employed to assemble the mock community. We also would like to thank Florian Leese, Arne Beermann, Cristina Hartmann-Fatu, and Marie Gutgesell for collecting and providing specimens. This study was supported by funding through the Canada First Research Excellence Fund. The funders had no role in study design, data collection, and analysis, decision to publish, or preparation of the manuscript. This work represents a contribution to the University of Guelph Food From Thought research program.

\section{AUTHORS' CONTRIBUTIONS}

B.B., V.E., T.B. and D.S. designed the experiments; B.B. and S.M. assembled the mock community; B.B. did the laboratory work; V.E. did the MiSeq experiment; B.B. and Z.C. analysed the data and built the bioinformatics pipeline; B.B. and D.S. wrote the manuscript, all authors contributed to the manuscript.

\section{PEER REVIEW}

The peer review history for this article is available at https://publons. com/publon/10.1111/2041-210X.13561.

\section{DATA AVAILABILITY STATEMENT}

Software ASHURE is deposited at Zenodo https://doi.org/10.5281/ zenodo.4450611 (Baloğlu \& Chen, 2021) and is available at Github under https://github.com/BBaloglu/ASHURE. Raw read data are available at the SRA under PRJNA627498 (ONT: Protocols A and B) and SRR9207930 (Illumina MiSeq).

\section{ORCID}

Bilgenur Baloğlu (iD) https://orcid.org/0000-0002-4434-5356

Zhewei Chen (iD https://orcid.org/0000-0002-7422-095X

Vasco Elbrecht iD https://orcid.org/0000-0003-4672-7099

Thomas Braukmann (iD https://orcid.org/0000-0002-2452-3776

Dirk Steinke iD https://orcid.org/0000-0002-8992-575X

\section{REFERENCES}

Adams, M., McBroome, J., Maurer, N., Pepper-Tunick, E., Saremi, N., Green, R. E., Vollmers, C., \& Corbett-Detig, R. B. (2019). One fly - one genome: Chromosome-scale genome assembly of a single outbred Drosophila melanogaster. BioRxiv, 866988. https://doi.org/10.1101/866988

Ankerst, M., Breunig, M. M., Kriegel, H. P., \& Sander, J. (1999). OPTICS: Ordering points to identify the clustering structure. SIGMOD Record (ACM Special Interest Group on Management of Data), 28(2), 49-60. https://doi.org/10.1145/304181.304187

Baloğlu, B., \& Chen, Z. (2021). ASHURE. Version 1.0.0. Zenodo. https:// doi.org/10.5281/zenodo.4450611

Batovska, J., Lynch, S. E., Cogan, N. O. I., Brown, K., Darbro, J. M., Kho, E. A., \& Blacket, M. J. (2018). Effective mosquito and arbovirus surveillance using metabarcoding. Molecular Ecology Resources, 18(1), 3240. https://doi.org/10.1111/1755-0998.12682

Benítez-Páez, A., Portune, K. J., \& Sanz, Y. (2016). Species-level resolution of $16 \mathrm{~S}$ rRNA gene amplicons sequenced through the MinION ${ }^{\mathrm{TM}}$ portable nanopore sequencer. GigaScience, 5(1), 1-9. https://doi. org/10.1186/s13742-016-0111-z 
Calus, S. T., ljaz, U. Z., \& Pinto, A. J. (2018). NanoAmpli-Seq: A workflow for amplicon sequencing for mixed microbial communities on the nanopore sequencing platform. GigaScience, 7(12), 1-16. https://doi. org/10.1093/gigascience/giy140

Clarke, J., Wu, H. C., Jayasinghe, L., Patel, A., Reid, S., \& Bayley, H. (2009). Continuous base identification for single-molecule nanopore DNA sequencing. Nature Nanotechnology, 4(4), 265-270. https://doi. org/10.1038/nnano.2009.12

Cornelis, S., Gansemans, Y., Vander Plaetsen, A. S., Weymaere, J., Willems, S., Deforce, D., \& Van Nieuwerburgh, F. (2019). Forensic triallelic SNP genotyping using nanopore sequencing. Forensic Science International: Genetics, 38, 204-210. https://doi.org/10.1016/j.fsigen. 2018.11.012

Deamer, D., Akeson, M., \& Branton, D. (2016). Three decades of nanopore sequencing. Nature Biotechnology, 34(5), 518. https://doi. org/10.1038/nbt.3423

Donoso, A., \& Valenzuela, S. (2018). In-field molecular diagnosis of plant pathogens: Recent trends and future perspectives. Plant Pathology, 67(7), 1451-1461. https://doi.org/10.1111/ppa.12859

Edgar, R. C. (2010). Search and clustering orders of magnitude faster than BLAST. Bioinformatics, 26(19), 2460-2461. https://doi.org/10.1093/ bioinformatics/btq461

Edgar, R. C., \& Flyvbjerg, H. (2015). Error filtering, pair assembly and error correction for next-generation sequencing reads. Bioinformatics, 31(21), 3476-3482. https://doi.org/10.1093/bioinformatics/btv401

Elbrecht, V., \& Leese, F. (2017). Validation and development of COI metabarcoding primers for freshwater macroinvertebrate bioassessment. Frontiers of Environmental Science, 5, 11.

Elbrecht, V., \& Steinke, D. (2019). Scaling up DNA metabarcoding for freshwater macrozoobenthos monitoring. Freshwater Biology, 64(2), 380-387. https://doi.org/10.1111/fwb.13220

Faucon, P., Trevino, R., Balachandran, P., Standage-Beier, K., \& Wang, $X$. (2017). High accuracy base calls in nanopore sequencing. ACM International Conference Proceeding Series, Part F1309, 12-16. https:// doi.org/10.1145/3121138.3121186

Hansen, S., Faye, O., Sanabani, S. S., Faye, M., Böhlken-Fascher, S., Faye, O., Sall, A. A., Bekaert, M., Weidmann, M., Czerny, C.-P., \& Abd El Wahed, A. (2018). Combination random isothermal amplification and nanopore sequencing for rapid identification of the causative agent of an outbreak. Journal of Clinical Virology, 106(July), 23-27. https:// doi.org/10.1016/j.jcv.2018.07.001

Harris, J. K., Sahl, J. W., Castoe, T. A., Wagner, B. D., Pollock, D. D., \& Spear, J. R. (2010). Comparison of normalization methods for construction of large, multiplex amplicon pools for nextgeneration sequencing. Applied and Environmental Microbiology, 76, 3863-3868.

Heeger, F., Bourne, E. C., Baschien, C., Yurkov, A., Bunk, B., Spröer, C., Overmann, J., Mazzoni, C. J., \& Monaghan, M. T. (2018). Long-read DNA metabarcoding of ribosomal RNA in the analysis of fungi from aquatic environments. Molecular Ecology Resources, 18(6), 15001514. https://doi.org/10.1111/1755-0998.12937

Hernández-Triana, L. M., Prosser, S. W., Rodríguez-Perez, M. A., Chaverri, L. G., Hebert, P. D. N., \& Ryan Gregory, T. (2014). Recovery of DNA barcodes from blackfly museum specimens (Diptera: Simuliidae) using primer sets that target a variety of sequence lengths. Molecular Ecology Resources, 14(3), 508-518. https://doi.org/10.1111/1755-0998. 12208

Ivanova, N. V., Dewaard, J. R., \& Hebert, P. D. N. (2006). An inexpensive, automation-friendly protocol for recovering high-quality DNA. Molecular Ecology Notes, 6(4), 998-1002. https://doi.org/10.1111/ j.1471-8286.2006.01428.x

Ivanova, N. V., Fazekas, A. J., \& Hebert, P. D. N. (2008). Semi-automated, membrane-based protocol for DNA isolation from plants. Plant Molecular Biology Reporter, 26, 186. https://doi.org/10.1007/s1110 5-008-0029-4
Jain, M., Fiddes, I. T., Miga, K. H., Olsen, H. E., Paten, B., \& Akeson, M. (2015). Improved data analysis for the MinION nanopore sequencer. Nature Methods, 12(4), 351-356. https://doi.org/10.1038/ nmeth.3290

Jain, M., Koren, S., Miga, K. H., Quick, J., Rand, A. C., Sasani, T. A., Tyson, J. R., Beggs, A. D., Dilthey, A. T., Fiddes, I. T., Malla, S., Marriott, H., Nieto, T., O'Grady, J., Olsen, H. E., Pedersen, B. S., Rhie, A., Richardson, H., Quinlan, A. R., ... Loose, M. (2018). Nanopore sequencing and assembly of a human genome with ultra-long reads. Nature Biotechnology, 36, 338-345. https://doi.org/10.1038/nbt.4060

Kafetzopoulou, L. E., Efthymiadis, K., Lewandowski, K., Crook, A., Carter, D., Osborne, J., Pullan, S. T. (2018). Assessment of metagenomic Nanopore and Illumina sequencing for recovering whole genome sequences of chikungunya and dengue viruses directly from clinical samples. Eurosurveillance, 23(50), https://doi.org/10.2807/1560-7917. ES.2018.23.50.1800228

Kimura, M. (1980). A simple method for estimating evolutionary rates of base substitutions through comparative studies of nucleotide sequences. Journal of Molecular Evolution, 16(2), 111-120. https://doi. org/10.1007/BF01731581

Kono, N., \& Arakawa, K. (2019). Nanopore sequencing: Review of potential applications in functional genomics. Development Growth and Differentiation, 61(5), 316-326. https://doi.org/10.1111/dgd.12608

Krehenwinkel, H., Pomerantz, A., Henderson, J. B., Kennedy, S. R., Lim, J. Y., Swamy, V., Shoobridge, J. D., Graham, N., Patel, N. H., Gillespie, R. G., \& Prost, S. (2019). Nanopore sequencing of long ribosomal DNA amplicons enables portable and simple biodiversity assessments with high phylogenetic resolution across broad taxonomic scale. GigaScience, 8(5), 1-16. https://doi.org/10.1093/gigascience/ giz006

Li, C., Chng, K. R., Boey, E. J. H., Ng, A. H. Q., Wilm, A., \& Nagarajan, N. (2016). INC-Seq: Accurate single molecule reads using nanopore sequencing. GigaScience, 5(1), https://doi.org/10.1186/s13742-016-0140-7

$\mathrm{Li}, \mathrm{H}$. (2018). Minimap2: Pairwise alignment for nucleotide sequences. Bioinformatics, 34(18), 3094-3100. https://doi.org/10.1093/bioin formatics/bty191

Li, H., \& Durbin, R. (2009). Fast and accurate short read alignment with Burrows-Wheeler transform. Bioinformatics, 25(14), 1754-1760. https://doi.org/10.1093/bioinformatics/btp324

Li, W., \& Godzik, A. (2006). Cd-hit: A fast program for clustering and comparing large sets of protein or nucleotide sequences. Bioinformatics, 22(13), 1658-1659. https://doi.org/10.1093/bioinformatics/btl158

Lim, N. K. M., Tay, Y. C., Srivathsan, A., Tan, J. W. T., Kwik, J. T. B., Baloğlu, B., Meier, R., \& Yeo, D. C. J. (2016). Next-generation freshwater bioassessment: eDNA metabarcoding with a conserved metazoan primer reveals species-rich and reservoir-specific communities. Royal Society Open Science, 3(11). https://doi.org/10.1098/rsos.160635

Loit, K., Adamson, K., Bahram, M., Puusepp, R., Anslan, S., Kiiker, R., Drenkhan, R., \& Tedersoo, L. (2019). Relative performance of MinION (Oxford Nanopore Technologies) versus Sequel (Pacific Biosciences) thirdgeneration sequencing instruments in identification of agricultural and forest fungal pathogens. Applied and Environmental Microbiology, 85(21), 1-20. https://doi.org/10.1128/AEM.01368-19

Loman, N. J., Quick, J., \& Simpson, J. T. (2015). A complete bacterial genome assembled de novo using only nanopore sequencing data. Nature Methods, 12(8), 733-735. https://doi.org/10.1038/nmeth. 3444

Ma, X., Stachler, E., \& Bibby, K. (2017). Evaluation of Oxford nanopore MinIONTM sequencing for $16 \mathrm{~S}$ rRNA microbiome characterization. BioRxiv, 099960.

Maaten, L. V. D., \& Hinton, G. (2014). Visualizing data using t-SNE. Journal of Machine Learning Research, 15, 3221-3245. https://doi.org/10.1007/ s10479-011-0841-3

Mafune, K. K., Godfrey, B. J., Vogt, D. J., \& Vogt, K. A. (2019). A rapid approach to profiling diverse fungal communities using the MinION ${ }^{\mathrm{TM}}$ 
nanopore sequencer. BioTechniques, 68(2), 72-78. https://doi.org/ 10.2144/btn-2019-0072

Martin, M. (2011). Cutadapt removes adapter sequences from highthroughput sequencing reads. EMBnet.journal, 17(1), 10-12. https:// doi.org/10.14806/ej.17.1.200

McKinney, W. (2010). Data structures for statistical computing in Python. In S. van der Walt, \& J. Millman (Eds.), Proceedings of the 9th Python in Science Conference. pp. 51-56.

McNaughton, A. L., Roberts, H. E., Bonsall, D., de Cesare, M., Mokaya, J., Lumley, S. F., Golubchik, T., Piazza, P., Martin, J. B., de Lara, C., Brown, A., Ansari, M. A., Bowden, R., Barnes, E., \& Matthews, P. C. (2019). Illumina and Nanopore methods for whole genome sequencing of hepatitis B virus (HBV). Scientific Reports, 9(1), 1-14. https:// doi.org/10.1038/s41598-019-43524-9

Menegon, M., Cantaloni, C., Rodriguez-Prieto, A., Centomo, C., Abdelfattah, A., Rossato, M., Bernardi, M., Xumerle, L., Loader, S., \& Delledonne, M. (2017). On site DNA barcoding by nanopore sequencing. PLoS One, 12(10), e0184741. https://doi.org/10.1371/ journal.pone.0184741

Mori, Y., \& Notomi, T. (2009). Loop-mediated isothermal amplification (LAMP): A rapid, accurate, and cost-effective diagnostic method for infectious diseases. Journal of Infection and Chemotherapy, 15, 62-69. https://doi.org/10.1007/s10156-009-0669-9

Nicholls, S. M., Quick, J. C., Tang, S., \& Loman, N. J. (2019). Ultra-deep, long-read nanopore sequencing of mock microbial community standards. GigaScience, 8(5), https://doi.org/10.1093/GIGASCIENCE

Parker, J., Helmstetter, A. J., Devey, D., Wilkinson, T., \& Papadopulos, A. S. T. (2017). Field-based species identification of closely-related plants using real-time nanopore sequencing. Scientific Reports, 7(1), 8345. https://doi.org/10.1038/s41598-017-08461-5

Piper, A. M., Batovska, J., Cogan, N. O. I., Weiss, J., Cunningham, J. P., Rodoni, B. C., \& Blacket, M. J. (2019). Prospects and challenges of implementing DNA metabarcoding for high-throughput insect surveillance. GigaScience, 8, 1-22. https://doi.org/10.1093/gigascience/ giz092

Pomerantz, A., Peñafiel, N., Arteaga, A., Bustamante, L., Pichardo, F., Coloma, L. A., Barrio-Amorós, C. L., Salazar-Valenzuela, D., \& Prost, S. (2018). Real-time DNA barcoding in a rainforest using nanopore sequencing: Opportunities for rapid biodiversity assessments and local capacity building. GigaScience, 7(4), 1-14. https://doi.org/10.1093/ gigascience/giy033

Quick, J., Ashton, P., Calus, S., Chatt, C., Gossain, S., Hawker, J., Nair, S., Neal, K., Nye, K., Peters, T., De Pinna, E., Robinson, E., Struthers, K., Webber, M., Catto, A., Dallman, T. J., Hawkey, P., \& Loman, N. J. (2015). Rapid draft sequencing and real-time nanopore sequencing in a hospital outbreak of Salmonella. Genome Biology, 16(1), 114. https://doi.org/10.1186/s13059-015-0677-2

Rang, F. J., Kloosterman, W. P., \& de Ridder, J. (2018). From squiggle to basepair: Computational approaches for improving nanopore sequencing read accuracy. Genome Biology, 19(1), 90. https://doi. org/10.1186/s13059-018-1462-9

Ratnasingham, S., \& Hebert, P. D. N. (2007). The barcode of life data system. Molecular Ecology Notes, 7, 355-364. https://doi.org/ 10.1111/j.1471-8286.2006.01678.x

Ratnasingham, S., \& Hebert, P. D. N. (2013). A DNA-based registry for all animal species: The Barcode Index Number (BIN) system. PLoS One, 8(7), e66213. https://doi.org/10.1371/journal.pone.0066213

Shepherd, B. A., Tanjil, M. R. E., Jeong, Y., Baloğlu, B., Liao, J., \& Wang, M. C. (2020). Ångström- and nano-scale pore-based nucleic acid sequencing of current and emergent pathogens. MRS Advances, 5(56), 2889-2906. https://doi.org/10.1557/adv.2020.402

Simpson, J. T., Workman, R. E., Zuzarte, P. C., David, M., Dursi, L. J., \& Timp, W. (2017). Detecting DNA cytosine methylation using nanopore sequencing. Nature Methods, 14(4), 407-410. https://doi. org/10.1038/nmeth.4184

Sović, I., Šikić, M., Wilm, A., Fenlon, S. N., Chen, S., \& Nagarajan, N. (2016). Fast and sensitive mapping of nanopore sequencing reads with GraphMap. Nature Communications, 7(1), 11307. https://doi.org/ 10.1038/ncomms11307

Sow, A., Brévault, T., Benoit, L., Chapuis, M.-P., Galan, M., Coeur d'acier, A., Delvare, G., Sembène, M., \& Haran, J. (2019). Deciphering hostparasitoid interactions and parasitism rates of crop pests using DNA metabarcoding. Scientific Reports, 9(1). https://doi.org/10.1038/ s41598-019-40243-z

Srivathsan, A., Baloğlu, B., Wang, W., Tan, W. X., Bertrand, D., Ng, A. H. Q., Boey, E. J. H., Koh, J. J. Y., Nagarajan, N., \& Meier, R. (2018). A MinION ${ }^{\mathrm{TM}}$-based pipeline for fast and cost-effective DNA barcoding. Molecular Ecology Resources, 18(5), 1035-1049. https://doi.org/ 10.1111/1755-0998.12890

Srivathsan, A., Hartop, E., Puniamoorthy, J., Lee, W. T., Kutty, S. N., Kurina, O., \& Meier, R. (2019). Rapid, large-scale species discovery in hyperdiverse taxa using 1D MinION sequencing. BMC Biology, 17(1), 1-20. https://doi.org/10.1186/s12915-019-0706-9

Staats, M., Arulandhu, A. J., Gravendeel, B., Holst-Jensen, A., Scholtens, I., Peelen, T., Prins, T. W., \& Kok, E. (2016). Advances in DNA metabarcoding for food and wildlife forensic species identification. Analytical and Bioanalytical Chemistry, 408, 4615-4630. https://doi. org/10.1007/s00216-016-9595-8

Steinke, D., Prosser, S. W. J., \& Hebert, P. D. N. (2016). DNA barcoding of marine metazoans. Methods in Molecular Biology, 1452, 155-168. https://doi.org/10.1007/978-1-4939-3774-5_10

Tedersoo, L., Tooming-Klunderud, A., \& Anslan, S. (2018). PacBio metabarcoding of Fungi and other eukaryotes: Errors, biases, and perspectives. New Phytologist, 217, 1370-1385. https://doi.org/10.1111/ nph.14776

Vaser, R., Sovic, I., Nagarajan, N., \& Mile, Š. (2017). Fast and accurate de novo genome assembly from long uncorrected reads. Genome Research, 1-10. https://doi.org/10.1101/gr.214270.116.5

Volden, R., Palmer, T., Byrne, A., Cole, C., Schmitz, R. J., Green, R. E., $\&$ Vollmers, C. (2018). Improving nanopore read accuracy with the R2C2 method enables the sequencing of highly multiplexed fulllength single-cell cDNA. Proceedings of the National Academy of Sciences of the United States of America, 115(39), 9726-9731. https:// doi.org/10.1073/pnas.1806447115

Voorhuijzen-Harink, M. M., Hagelaar, R., van Dijk, J. P., Prins, T. W., Kok, E. J., \& Staats, M. (2019). Toward on-site food authentication using nanopore sequencing. Food Chemistry: $X, 2$, https://doi. org/10.1016/j.fochx.2019.100035

Walt, S. V. D., Colbert, S. C., \& Varoquaux, G. (2011). The NumPy array: A structure for efficient numerical computation. Computing in Science \& Engineering, 13(2), 22-30. https://doi.org/10.1109/MCSE.2011.37

\section{SUPPORTING INFORMATION}

Additional supporting information may be found online in the Supporting Information section.

How to cite this article: Baloğlu B, Chen Z, Elbrecht V,

Braukmann T, MacDonald S, Steinke D. A workflow for accurate metabarcoding using nanopore MinION sequencing. Methods Ecol Evol. 2021;12:794-804. https://doi. org/10.1111/2041-210X.13561 